\title{
ESTUDO RETROSPECTIVO DE EXAMES HISTOPATOLÓGICOS ESPLÊNICOS NA ROTINA LABORATORIAL DO HOSPITAL ESCOLA VETERINÁRIO UNIFAJ - DE 2015 A 2020.
}

\author{
Thaís van den Broek Campanelli ${ }^{1}$ \\ Débora Lucinda Alves ${ }^{1}$ \\ Stefanie Regina Lima ${ }^{1}$ \\ Gabriele Braga ${ }^{1}$ \\ Adriano Jaskonis Dall'Olio ${ }^{2}$
}

\begin{abstract}
RESUMO
O baço canino exerce diversas funções, dentre elas linfáticas, hematopoiéticas, imunológicas e circulatórias, sendo que suas características morfológicas e funcionais facilitam a ocorrência de lesões neoplásicas e não neoplásicas. Este trabalho teve como objetivo realizar um estudo retrospectivo dos resultados histopatológicos esplênicos, oriundos do Laboratório do Hospital Escola Veterinário do Centro Universitário de Jaguariúna (HEV- UNIFAJ) - Jaguary no período de 2015 a 2020. Foram analisadas 44 amostras esplênicas, e pelos achados histopatológicos 15/44 (34,1\%) se tratavam de neoplasias, 28/44 (63,6\%) representaram alterações não neoplásicas, enquanto que $1 / 44$ (2,3\%) foi diagnosticado como sítio metastático. Levando em consideração a prevalência das alterações não neoplásica, juntamente com as novas abordagens terapêuticas realizadas em medicina humana, a Medicina Veterinária poderia levar em conta tais fatores na elaboração de modernos planejamentos cirúrgicos e atuais escolhas de tratamentos.
\end{abstract}

Palavras-chave: neoplasia, baço, abordagens terapêuticas, histopatológico.

\section{RETROSPECTIVE STUDY OF SPLENIC HISTOPATHOLOGICAL EXAMINATIONS IN THE LABORATORY ROUTINE OF THE UNIFAJ VETERINARY SCHOOL HOSPITAL - FROM 2015 TO 2020.}

\begin{abstract}
The canine spleen has several functions, including lymphatic, hematopoietic, immunological and circulatory, and its morphological and functional characteristics facilitate the occurrence of neoplastic and non-neoplastic lesions. This work aims to carry out a retrospective study of splenic histopathological results, from the Laboratory of the Veterinary School Hospital of the Centro Universitário de Jaguariúna (HEV-UNIFAJ) - Jaguary in the period from 2015 to 2020. 44 splenic samples were analyzed, and the histopathological findings 15 / 44 (34.1\%) were neoplasms, 28/44 (63.6\%) represented non-neoplastic alterations, while 1/44 (2.3\%) was diagnosed as a metastatic site. Taking into account the prevalence of non-neoplastic alterations, together with the new therapeutic approaches in human medicine, Veterinary Medicine could take such factors into account in the elaboration of modern surgical plans and current treatment choices.
\end{abstract}

Keywords: neoplasm, spleen, therapeutic approaches, histopathological.

\footnotetext{
${ }^{1}$ Unifaj*Contato principal para correspondência editorial. thaisvdbroek@gmail.com ${ }^{2}$ USP, jaskonis@hotmail.com

Campanelli TVDB, Alves DL, Lima SR, Braga G, Dall'Olio AJ. Estudo retrospectivo de exames histopatológicos esplênicos na rotina laboratorial do hospital Escola Veterinário UNIFAJ - de 2015 a 2020. Vet. e Zootec. 2021; v28: 001-007.
} 


\section{ESTUDIO RETROSPECTIVO DE EXÁMENES HISTOPATOLÓGICOS ESPLÉNICOS EN LA RUTINA DE LABORATORIO DEL HOSPITAL ESCOLAR VETERINARIO UNIFAJ - DESDE 2015 A 2020.}

\section{RESUMEN}

El bazo canino ejerce diversas funciones, entre ellas linfáticas, hematopoyéticas, inmunológicas y circulatorias, siendo que sus características morfológicas y funcionales facilitan la occurencia de lesiones neoplásicas y no neoplásicas. Este trabajo tuvo como objetivo realizar un estudio retrospectivo de los resultados histopatológicos esplénicos, oriundos del Laboratorio del Hospital Escuela Veterinaria del Centro Universitário de Jaguariúna (HEV-UNIFAJ) - Jaguary en el período de 2015 a 2020. Se analizaron 44 muestras esplénicas, en los hallazgos histopatológicos 15/44 (34,1\%) se trataban neoplasias, $28 / 44(63,6 \%)$ representaron alteraciones no neoplásicas, mientras que 1/44 (2,3\%) fue diagnosticado como sitio metastásico. Teniendo en cuenta la prevalencia de las alteraciones no neoplásicas, junto con los nuevos enfoques terapéuticos realizados em la medicina humana, la Medicina Veterinaria podría tener en cuenta tales factores en la elaboración de modernos planes quirúrgicos y actuales opciones de tratamiento.

Palabras clave: neoplasia, bazo, abordajes terapéuticos, histopatológicos.

\section{INTRODUÇÃO}

O baço canino é um órgão anatomicamente e funcionalmente composto por uma polpa vermelha, altamente vascularizada, a qual atua removendo eritrócitos anormais, imunocomplexos e bactérias, e uma polpa branca, constituída por tecido linfóide, responsável pela fagocitose de antígenos (1), além disso atua como importante reservatório de hemácias e plaquetas (2). Dentre suas funções, linfáticas, hematopoiéticas, imunológicas e circulatórias, a hematopoiética é considerada a principal atividade observada durante a vida fetal canina, enquanto na vida adulta todas as funções são tidas como relevantes $(3,4)$. Na sua ausência, a medula óssea vermelha e o fígado se tornam responsáveis pela remoção de eritrócitos anormais, já que são órgãos ricos em macrófagos, ademais o sistema linfático também assume parte de suas funções (5).

Segundo Lipowitz e Blue (6), as características morfológicas e funcionais do baço facilitam a ocorrência de lesões neoplásicas (benignas e malignas) e não neoplásicas (inflamatórias, circulatórias, hematológicas, hiperplásicas), em função de sua composição parenquimatosa. Estudos apontam que cães são mais acometidos por alterações neoplásicas do que alterações não neoplásicas e, observa-se maior incidência de neoplasia em cães machos de meia idade a idosos $(3,7)$.

As neoplasias esplênicas podem ser classificadas em: primárias - benignas ou malignas e secundárias - por traumas ou sítios metastáticos (8). As neoplasias esplênicas primárias classificadas como benignas incluem: o hemangioma, lipoma, fibroma e linfangioma esplênico (9). Dentre as neoplasias primárias malignas, o hemangiossarcoma (HSA), é considerado o de maior ocorrência (10), seguidos dos linfomas e sarcomas, tais como fibrossarcoma, leiomiossarcoma e sarcomas histiocíticos (9). Sítios metastáticos esplênicos são raros, e quando ocorrem estão associados a tumores primários como, sarcomas e carcinomas em mamas, pulmões, pâncreas ou melanomas $(9,11,12)$.

As alterações clínicas associadas às neoplasias esplênicas são inespecíficas e incluem anorexia, letargia, vômito e distensão abdominal e, em alguns casos o hemoabdome espontâneo. (8). Deste modo, ressalta-se a importância da realização do teste de triagem 
padrão para avaliação focalizada com sonografia para trauma abdominal (AFAST), a fim de identificar efusão abdominal (13). O exame ultrassonográfico abdominal, deve ser realizado para avaliação morfológica e estrutural do órgão, a fim de se identificar neoformações, todavia, a classificação desta e sua confirmação diagnóstica devem ser realizadas pelo exame histopatológico - percutâneo ou incisional $(1,14)$. Tem-se ainda como métodos diagnósticos a tomografia computadorizada e o raio-X torácicos e abdominais, os quais devem ser considerados para identificação da neoformação e estadiamento em casos com potencial metastáticos (8).

A escolha do tratamento deve ser de acordo com a necessidade e, priorizar sempre que possível, a permanência total ou parcial do órgão $(8,15)$. A esplenectomia total em cães pode predispor a ocorrência de infecções microbianas, parasitismo de eritrócitos e sepse, além de diminuir a tolerância ao exercício e gerar uma resposta inadequada à hipóxia e ao choque (16). Ademais, complicações pós-cirúrgicas como hemorragia, trombose aguda da veia porta e arritmias foram descritas por Tobias e Johnston (4).

Na medicina humana a realização da esplenectomia total é tida como último recurso, visto que pacientes esplenectomizados apresentam maiores taxas de infecções (17). Desta forma, tanto em humanos quanto em animais, a esplenectomia parcial e novas abordagens tem sido cada vez mais estudadas e aplicadas (18). As alternativas terapêuticas podem ser consideradas promissoras e variam desde eletrocirurgia, laparoscopia, autoimplantes de baço $(19,20,21)$ até tecnologias moleculares e a edição genética utilizando o Conjunto de Repetições Palindrômicas Curtas Regularmente Espaçadas (CRISPR/Cas9) a fim de preservar o órgão e diminuir as complicações causadas por procedimentos cirúrgicos e quimioterapia (22).

Este trabalho teve como objetivo realizar um estudo retrospectivo dos resultados histopatológicos esplênicos, oriundos do Laboratório de diagnóstico veterinário do Hospital Escola Veterinário do Centro Universitário de Jaguariúna (HEV- UNIFAJ) - Jaguary, a fim de divulgar esses resultados para que sirvam de referência para novas propostas e abordagens cirúrgicas e terapêuticas.

\section{MATERIAIS E MÉTODOS}

Foi realizado um levantamento dos resultados histopatológicos de amostras esplênicas, no período de 2015 a 2020 . Essas amostras analisadas foram todas oriundas do Laboratório de diagnóstico veterinário do Hospital Escola Veterinário do Centro Universitário de Jaguariúna (HEV- UNIFAJ) -Jaguary. Para compilação dos dados, considerou-se, além dos resultados histopatológicos, o sexo e a idade desses pacientes.

\section{RESULTADOS}

Durante o intervalo de tempo de 2015 a 2020, o Laboratório de diagnóstico veterinário do Hospital Escola Veterinário - HEV - do Centro Universitário de Jaguariúna, Jaguariúna-SP - Jaguary efetuou um total de 44 análises histopatológicas de amostras de baço canino.

Dentre os achados histopatológicos, $15 / 44(34,1 \%)$ se tratavam de acometimento neoplásico, 28/44 (63,6\%) foram classificados como não neoplásicos enquanto que 1/44 $(2,3 \%)$ foi diagnosticado como sítio metastático. As tabelas 1,2 e 3 representam, respectivamente, os resultados histopatológicos neoplásicos, não neoplásicos e por sítio metastático encontrados neste estudo, assim como a faixa etária (idade média foi de 9,4 anos) e o sexo dos cães acometidos. Os machos apresentaram maior prevalência, representando $59,1 \%(26 / 44)$, enquanto $40,9 \%(18 / 44)$ eram fêmeas.

Campanelli TVDB, Alves DL, Lima SR, Braga G, Dall'Olio AJ. Estudo retrospectivo de exames histopatológicos esplênicos na rotina laboratorial do hospital Escola Veterinário UNIFAJ - de 2015 a 2020. Vet. e Zootec. 2021; v28: 001-007. 
Tabela 1. Resultados histológicos neoplásicos.

\begin{tabular}{lcccccc}
\hline \multirow{2}{*}{ Alterações neoplásicas } & \multirow{2}{*}{$\mathbf{N}^{\mathbf{0}}$} & \multirow{2}{*}{$\begin{array}{c}\text { Idade média } \\
\text { (anos) }\end{array}$} & \multicolumn{2}{c}{ Sexo } \\
\hline Hemangioma & 5 & 33,3 & 8,6 & 2 & $\mathrm{~F}$ \\
Hemangiossarcoma & 4 & 26,7 & 10 & 0 & 4 \\
Neoplasia maligna pouco diferenciada & 2 & 13,3 & 10,5 & 0 & 2 \\
Mielolipoma & 2 & 13,3 & 6 & 2 & 0 \\
Linfoma esplênico nodular & 1 & 6,7 & 11 & 0 & 1 \\
Sarcoma estromal esplênico & \multicolumn{1}{c}{ TOTAL } & 1 & 6,7 & 10 & 1 & 0 \\
\hline \multicolumn{2}{c}{$\mathbf{1 5}$} & $\mathbf{1 0 0}$ & $\mathbf{9 , 4}$ & $\mathbf{5}$ & $\mathbf{1 0}$ \\
\hline
\end{tabular}

Tabela 2. Resultados histológicos não neoplásicos.

\begin{tabular}{|c|c|c|c|c|c|}
\hline \multirow{2}{*}{ Alterações não neoplásicas } & \multirow{2}{*}{$\mathbf{N}^{\mathbf{o}}$} & \multirow{2}{*}{$\%$} & \multirow{2}{*}{$\begin{array}{l}\text { Idade média } \\
\text { (anos) }\end{array}$} & \multicolumn{2}{|c|}{ Sexo } \\
\hline & & & & $\mathrm{M}$ & $\mathrm{F}$ \\
\hline Hiperplasia de tecido linfoide & 15 & 53,6 & 9,4 & 9 & 6 \\
\hline Hiperplasia de polpa vermelha & 3 & 10,7 & 9,7 & 3 & 0 \\
\hline Hiperplasia nodular & 3 & 10,7 & 9 & 3 & 0 \\
\hline Necrose esplênica & 2 & 7,1 & 10 & 1 & 1 \\
\hline Rarefação de tecido linfoide & 2 & 7,1 & 11 & 1 & 1 \\
\hline Congestão & 1 & 3,6 & 5 & 0 & 1 \\
\hline Esplenite crônica & 1 & 3,6 & 8 & 1 & 1 \\
\hline Infarto & 1 & 3,6 & 11 & 1 & 1 \\
\hline TOTAL & 28 & 100 & 9,1 & 19 & 9 \\
\hline
\end{tabular}

Tabela 3. Resultado histológico de sítio metastático.

\begin{tabular}{lcccccc}
\hline \multirow{2}{*}{ Diagnóstico histopatológico } & \multirow{2}{*}{$\mathbf{N}^{\mathbf{0}}$} & $\mathbf{\%}$ & $\begin{array}{c}\text { Idade média } \\
(\text { anos) }\end{array}$ & & \multicolumn{2}{c}{ Sexo } \\
\hline Hepatocarcinoma & 1 & 1 & 12 & 0 & $\mathrm{~F}$ \\
\hline \multicolumn{1}{c}{ TOTAL } & & & & & & \\
\hline
\end{tabular}

\section{DISCUSSÃO}

Esse estudo retrospectivo contempla o diagnóstico histopatológico de 44 amostras esplênicas caninas, submetidas ao Laboratório de diagnóstico veterinário do Hospital Escola Veterinário do Centro Universitário de Jaguariúna (HEV- UNIFAJ) - Jaguary. As amostras analisadas tinham histórico de serem provenientes de esplenectomias totais, decorrentes de pacientes com quadros clínicos/cirúrgicos de urgência/emergência, procedimento rotineiro no planejamento cirúrgico de afecções neoplásicas e não neoplásicas com hemoabdome espontâneo. Fossum (8) sugere que a esplenectomia parcial é preferida sempre que possível. Johnson et al. (16), Tilson (2) e Tobias e Johnston (4) em seus estudos relataram que os cães apresentaram determinadas consequências e vulnerabilidades com a esplenectomia total e, em alguns casos, como trombocitoses, hemorragias e obstruções vasculares, são tidas como potencialmente fatais.

Os resultados obtidos nas análises histopatológicas não divergiram dos que foram observados por Bettini et al. (10), Dionísio (9) e Figueiredo et al (14) no que se refere a 
incidência neoplásica, sítios metastáticos e alterações não neoplásicas, respectivamente. As alterações esplênicas não neoplásicas foram consideradas como o resultado mais frequente dentre as amostras histopatológicas, representando $63,6 \%$. Tal resultado difere do descrito por Bandinelli et al. (3) e Xavier (7), já que as alterações não neoplásicas representaram, respectivamente, $30,6 \%$ e $40 \%$ dos casos em seus estudos; podendo estar associado com a quantidade total das amostras analisadas ou a alta incidência de acometimento hemoparasitário. Segundo o levantamento de Oliveira, Costa e Abreu (23) 58,20\% dos cães foram positivos para Ehrlichia canis na região de Campinas, sendo Jaguariúna a segunda cidade com maior número de cães acometidos (13,11\%).

A realização da esplenectomia total vem decrescendo nitidamente na medicina humana, em virtude do reconhecimento da potencialidade e aumento de taxas da infecção fulminante pós esplenectomia (IFPE) (17), além de novas orientações em relação a tratamentos, como a utilização de eletrocirurgias, que promovem fácil hemostasia e, consequentemente menor tempo cirúrgico e algia pós operatória; oclusões vasculares com clipes hemostáticos, bisturi ultra-sônico ativado e selador vascular bipolar eletrotérmico, os quais tem sido avaliados em cães para melhor hemostasia durante o procedimento de esplenectomia $(19,24)$; auto-implante em casos que a esplenectomia se torna inevitável (21); além do uso da biologia molecular, como a CRISPR/Cas9 (22). Levando em consideração as alterações esplênicas não neoplásica, relatadas como de maior prevalência neste estudo, juntamente com as possíveis complicações de esplenectomia total e as novas abordagens terapêuticas realizadas na medicina humana, a Medicina Veterinária poderia levar em conta tais fatores na elaboração de modernos planejamentos cirúrgicos e atuais escolhas de tratamentos.

\section{CONCLUSÃO}

Ainda que o baço desempenhe maior papel na vida fetal, este não perde sua importância nas fases adulta e senil, uma vez que continua a exercer suas funções, dentre elas a defesa do organismo, papel hematológico e reservatório de células, sendo fundamental, antes de optar por sua retirada total, avaliar a real necessidade e os riscos envolvidos, e assim ponderar sobre outras possibilidades cirúrgicas e terapêuticas disponíveis; muitas dessas já descritas e utilizadas na medicina humana, avaliar possíveis adaptações e aplicações na medicina veterinária.

\section{REFERÊNCIAS}

1. Jericó MM, Andrade Neto JP, Kogika MM. Tratado de medicina interna de cães e gatos. Rio de Janeiro: Guanabara Koogan; 2015.

2. Tilson M. Spleen. In: Slatter D. Textbook of small animal surgery. 3a ed. Philadelphia: Elsevier Science; 2003. p. 1046-62.

3. Bandinelli MB, Pavarini SP, Oliveira EC, Gomes DC, Cruz CEF, Driemeier D. Estudo retrospectivo de lesões em baços de cães esplenectomizados: 179 casos. Pesqui Vet Bras [Internet]. 2011 [citado 20 Out 2020];31:697-701. Disponível em: https://www.scielo.br/scielo.php?pid=S0100-

736X2011000800011\&script $=$ sci_abstract\&tlng $=\mathrm{pt}$

4. Tobias KM, Johnston SA. Veterinary surgery: small animal. Riverpot Lane: Elsevier Saunders; 2012.

Campanelli TVDB, Alves DL, Lima SR, Braga G, Dall'Olio AJ. Estudo retrospectivo de exames histopatológicos esplênicos na rotina laboratorial do hospital Escola Veterinário UNIFAJ - de 2015 a 2020 . Vet. e Zootec. 2021; v28: 001-007. 
5. Junqueira LC, Carneiro J. Histologia básica. 10a ed. Rio de Janeiro: Guanbara Koogan; 2004. Sistema imunitário e órgãos linfáticos; p. 276-80.

6. Lipowitz AJ, Blue J. Baço. In: Slatter DH. Manual de cirurgia de pequenos animais. São Paulo: Manole; 1998. p. 1143-59.

7. Xavier DM. Alterações esplênica em cães: estudo retrospectivo de 43 casos [monografia]. Botucatu: Instituto Bioethicus; 2013.

8. Fossum TW. Cirurgia de pequenos animais. Rio de Janeiro: Elsevier; 2014.

9. Dionísio MIM. Prevalência da doença esplénica em cães e sobrevivência após esplenectomia: estudo retrospectivo [dissertação]. Lisboa: Faculdade de Medicina Veterinária, Universidade de Lisboa; 2016.

10. Bettini G, Mandrioli L, Brunetti B, Marcato PS. Canine splenic pathology: a retrospective study of 109 surgical samples, with special emphasis on fibrohistiocytic nodules. Eur J Vet Pathol [Internet]. 2001 [citado 10 Set 2020];7:101-9. Disponível em: https://www.vetjournal.it/images/archive/pdf_riviste/2684.pdf

11. Lopes BT. Esplenectomias: indicações, complicações e alternativas [trabalho de conclusão de curso]. Florianópolis: Faculdade de Medicina, Universidade Federal de Santa Catarina; 2003.

12. Dalek CR, De Nardi AB. Oncologia em cães e gatos. Rio de Janeiro: Roca; 2016.

13. Boysen SR, Lisciandro GR. The use of ultrasound for dogs and cats in the emergency room AFAST and TFAST. Vet Clin North Am Small Anim Pract. 2013;43:773-97.

14. Figueiredo RS, Muramoto C, Fontes TN, Meneses IDS, Cardoso PGS, Vieira Filho CHC, et al. Lesions in 224 spleens of splenectomized dogs and evalution of alternative techniques for previous microscopic diagnosis. Pesqui Vet Bras [Internet]. 2019 [citado 15 Nov 2020];39:622-9. Disponível em: https://www.scielo.br/scielo.php?script=sci_arttext\&pid=S0100-736X2019000800622

15. Sousa JUJO. Esplenopatias cirúrgicas em cães, estudo retrospectivo num hospital de referência na área da Grande Lisboa [dissertação]. Lisboa: Faculdade de Medicina Veterinária, Universidade Lusófona de Humanidades e Tecnologias; 2012.

16. Johnson KA, Powers BE, Withrow SJ, Sheetz MJ, Curtis CR, Wrigley RH. Splenomegaly in dogs - predictors of neoplasia and survival after splenectomy. J Vet Intern Med. 1989;3:160-6.

17. Marques RG, Petroianu A. Infecção fulminante pós esplenectomia. Arq Gastroenterol [Internet]. 2003 [citado 17 Nov 2020];40:47-54. Disponível em: https://www.scielo.br/scielo.php?script=sci_arttext\&pid=S0004-28032003000100011

18. Breton AM. Spleen physiology and the treatment of common diseases. Greenwood Village: American College of Veterinary Internal Medicine; 2012.

Campanelli TVDB, Alves DL, Lima SR, Braga G, Dall'Olio AJ. Estudo retrospectivo de exames histopatológicos esplênicos na rotina laboratorial do hospital Escola Veterinário UNIFAJ - de 2015 a 2020. Vet. e Zootec. 2021; v28: 001-007. 
19. Trindade RM, Grazziotin UR. Eletrocirurgia: sistemas mono e bipolar em cirurgia videolaparoscópica. Acta Cir Bras [Internet]. 1998 [citado 28 Out 2020];13(3):1-17. Disponível em: https://www.scielo.br/scielo.php?script=sci_arttext\&pid=S010286501998000300010

20. Stedile R, Beck CAC, Schiochet F, Ferreira MP, Oliveira ST, Martens FB, et al. Laparoscopic versus open splenectomy in dogs. Pesqui Vet Bras [Internet]. 2009 [citado 3 Nov 2020];29:653-60. Disponível em: https://www.scielo.br/pdf/pvb/v29n8/a09v29n8.pdf

21. Cardoso DL, Cardoso Filho FA, Cardoso AL, Gonzaga ML, Grande AJ. Autoimplante esplênico deve ser considerado para pacientes submetidos à esplenectomia total por trauma? Rev Col Bras Cir [Internet]. 2018 [citado 14 Out 2020];45:1-9. Disponível em: https://www.scielo.br/scielo.php?pid=S0100-

$69912018000300300 \&$ script $=$ sci_arttext\&tlng $=$ pt

22. Khalaf L, Janowicz K, Konwinska MD, Hutchings G, Dompe C, Moncrieff L, et al. CRISPR/Cas9 in cancer immunotherapy: animal models and human clinical trials. Genes [Internet]. 2020 [citado 10 Dez 2020];11:1-19. Disponível em: https://www.mdpi.com/2073-4425/11/8/921

23. Oliveira A, Costa DP, Abreu RT. Diagnóstico de erliquiose e anaplasmose utilizando snap test rapido em cães na região de Campinas [trabalho de conclusão de curso]. Jaguariúna: Faculdade de Medicina Veterinária, Centro Universitário de Jaguariúna; 2015.

24. Royals SR, Ellison GW, Adin CA, Wheeler JL, Sereda CW, Krotscheck U. Use of an ultrasonically activated scalpel for splenectomy in 10 dogs with naturally occurring splenic disease. Vet Surg. 2005;34:174- 8.

Recebido em: 03/02/2021

Aceito em: 17/09/2021

Campanelli TVDB, Alves DL, Lima SR, Braga G, Dall'Olio AJ. Estudo retrospectivo de exames histopatológicos esplênicos na rotina laboratorial do hospital Escola Veterinário UNIFAJ - de 2015 a 2020 . Vet. e Zootec. 2021; v28: 001-007. 